\title{
Diphtérie respiratoire mortelle chez une personne en visite au Canada
}

\author{
Scott Cholewa MA BScS DPA, Fareen Karachiwalla MD MSP, Sarah E. Wilson MD MSc, Jeya Nadarajah MD MSc, \\ Julianne V. Kus PhD MSc
}

- Citation : CMAJ 2021 January 4;193:E19-22. doi : 10.1503/cmaj.200707-f

Voir la version anglaise de l'article ici : www.cmaj.ca/lookup/doi/10.1503/cmaj.200707

$\mathbf{U}$

ne personne indienne de 69 ans en visite au Canada est venue consulter à l'hôpital pour une détresse respiratoire aiguë 48 heures après son arrivée au pays. Elle a signalé qu'au moment de son départ de l'Inde, elle avait des maux de gorge, la voix enrouée et une toux sèche, et qu'en 48 heures, elle avait développé un essoufflement, de la fièvre et de la diaphorèse. Cette personne était autrement en santé et n'avait pas été en contact avec des personnes malades; elle n'était toutefois pas à jour dans ses vaccins.

Elle a d'abord consulté une clinique de télémédecine, qui lui a conseillé de se rendre immédiatement au service des urgences. L'examen physique réalisé sur place a révélé la présence d'un léger œdème au cou. Les nœuds lymphatiques n'étaient pas palpables, et aucun stridor n'était audible. La personne a dit avoir l'impression que quelque chose était coincé dans sa gorge, mais aucune obstruction n'a été constatée. Une radiographie du cou a mis en évidence un important œdème des tissus mous prévertébraux, et une radiographie des poumons a montré une consolidation au lobe inférieur gauche. Un diagnostic d'influenza probable et de pneumonie bactérienne concomitante a été posé, et de la ceftriaxone et de l'oseltamivir ont été prescrits, conformément aux lignes directrices locales. La personne a été hospitalisée, et son état s'est détérioré dans les 48 heures suivantes, au point de présenter une insuffisance respiratoire hypoxémique et un syndrome de détresse respiratoire aiguë, qui s'est avéré mortel.

L'hémoculture, la culture de virus respiratoire (adénovirus, influenza $A$ et $B$, métapneumovirus humain, virus respiratoire syncytial) à partir d'échantillons nasopharyngés ainsi que la recherche d'antigènes urinaires de Legionella ont donné des résultats négatifs. Après le décès de la personne, nous avons reçu les résultats de la culture des sécrétions endotrachéales prélevées au moment de l'intubation, qui faisaient état d'une légère croissance pure de bactéries corynéformes. Les bactéries corynéformes ou diphtériques obtenues à la culture de sécrétions provenant de milieux non stériles sont généralement considérées comme des bactéries commensales de faible signification clinique. Or, comme il s'agissait ici d'une culture pure, nous avons mené d'autres examens.

\section{POINTS CLÉS}

- Bien qu'elle soit rare, la diphtérie respiratoire classique produisant des toxines survient parfois au Canada. Elle devrait faire partie du diagnostic différentiel chez les personnes aux prises avec une affection respiratoire aiguë qui sont récemment arrivées d'un pays où la diphtérie est endémique ou qui ne sont pas à jour dans leurs vaccins.

- La diphtérie respiratoire est une urgence médicale nécessitant l'administration d'antitoxines et d'antimicrobiens.

- La prise en charge de la diphtérie par les autorités régionales de santé publique comprend la recherche des contacts et peut inclure une prophylaxie postexposition et une immunisation.

- La diphtérie toxigène est une maladie évitable par la vaccination. Les personnes nées au Canada, celles nouvellement arrivées au pays et celles prévoyant voyager dans un pays où la diphtérie est toujours présente devraient s'assurer d'avoir reçu tous leurs vaccins.

Une spectrométrie de masse par désorption-ionisation laser assistée par matrice et à analyseur de temps de vol (MALDI-TOF MS) a révélé que la bactérie en cause était Corynebacterium diphtheriae. Ce microorganisme étant rarement identifié dans la plupart des laboratoires canadiens, nous avons envoyé l'isolat aux laboratoires de référence de la province et du pays pour une nouvelle identification et une analyse de toxines. Un test d'amplification en chaîne par polymérase (PCR) réalisé par le Laboratoire national de microbiologie a démontré que la bactérie portait le gène tox, qui la rendait capable de produire une toxine diphtérique. Alors que nous attendions la confirmation qu'il s'agissait hors de tout doute d'une souche produisant une toxine diphtérique (par le test Elek modifié), le Laboratoire nous a informés que la majorité des isolats porteurs du gène tox sont des producteurs de toxine ${ }^{1}$. À la lumière de cette information, des antécédents cliniques de la personne décédée et des renseignements épidémiologiques disponibles, nous avons entrepris une recherche des contacts. La production de toxine diphtérique a été confirmée 6 jours après la PCR. 
La recherche des contacts a été possible grâce à une collaboration entre le bureau régional de santé publique et le service de prévention et de contrôle des infections de l'hôpital, ainsi qu'au soutien de Santé publique Ontario et de l'Agence de la santé publique du Canada. Elle a permis de repérer 4 membres du ménage et 7 professionnels de la santé ayant potentiellement été exposés à des sécrétions oropharyngées alors qu'ils ne portaient pas d'équipement de protection individuelle. Ces contacts ont tous été évalués à la recherche de signes et symptômes d'infection. Ils ont subi des écouvillonnages nasopharyngés et de gorge en vue de cultures bactériennes, ont reçu une chimioprophylaxie antimicrobienne (érythromycine), ont été vaccinés si leur couverture vaccinale n'était pas à jour, et ont été exclus des milieux scolaires et de soins jusqu'à l'obtention des résultats des cultures. Aucun cas de transmission secondaire n'a été relevé.

\section{Discussion}

L'augmentation des voyages internationaux et la réticence croissante à la vaccination multiplient les possibilités d'urgences de santé publique - attribuables par exemple à la diphtérie respiratoire produisant des toxines - ou associées à des troubles médicaux rares au Canada. Le cas présenté ici rappelle aux professionnels de la santé qu'il importe d'envisager le diagnostic de diphtérie, surtout chez les personnes arrivant d'une région endémique et présentant une infection respiratoire aiguë, et de promouvoir la vaccination tout au long de la vie.

\section{Épidémiologie}

La diphtérie est rare au Canada, grâce à l'efficacité des programmes de vaccination mis en place dans les années 1920. Au pays, l'incidence de la diphtérie toxigène est passée d'environ 100 cas pour 100000 personnes en 1924 à 0,03 cas pour 100000 personnes ( $n=10$ cas) en $2017^{2}$. La plus récente infection mortelle chez un résident canadien est survenue en 2010, et le dernier cas de diphtérie respiratoire toxigène signalé en Ontario remonte à 1995 .

\section{Présentation clinique et prise en charge en phase aiguë} La diphtérie, qui peut prendre diverses formes, s'accompagne d'une vaste gamme de manifestations cliniques. Elle est catégorisée en fonction du site d'infection (infection cutanée ou respiratoire) et de la production de toxines. La diphtérie respiratoire est causée par des souches toxigènes de la bactérie à Gram positif $C$. diphtheriae ou, plus rarement, par d'autres espèces toxigènes (C. ulcerans ou $C$. pseudotuberculosis) ${ }^{4}$. Les premiers symptômes de la diphtérie pharyngée ou amygdalienne sont généralement la fièvre, une pharyngite, une voix enrouée et une inflammation des nœuds lymphatiques cervicaux; l'infection peut progresser jusqu'à une obstruction des voies respiratoires supérieures et une détresse respiratoire aiguë ${ }^{4}$. Deux des signes classiques de diphtérie respiratoire toxigène sont la présence d'une membrane grisâtre sur les amygdales et une inflammation importante de l'uvule, des amygdales, des nœuds lymphatiques cervicaux et de la partie antérieure du cou et de la région sousmaxillaire, rappelant un cou de taureau ${ }^{5}$.
Les complications associées à la diffusion des toxines comprennent la neuropathie périphérique et la myocardite ${ }^{6}$. On estime que le taux de létalité de la diphtérie respiratoire classique est $5 \%-10 \%$. La toxigénicité d'une souche est établie en fonction de la présence du gène tox, confirmée au moyen d'une $\mathrm{PCR}$, et de l'expression d'une toxine diphtérique, confirmée au moyen du test Elek modifié. La diphtérie respiratoire toxigène est une urgence médicale nécessitant l'administration d'antitoxines diphtériques et d'antimicrobiens, sans attendre une confirmation en laboratoire. Une assistance respiratoire et un suivi attentif des fonctions cardiaques et neurologiques sont essentiels. Pour obtenir des antitoxines diphtériques, les professionnels de la santé doivent communiquer avec les bureaux de santé publique. En Ontario, la distribution est coordonnée par le ministère de Santé, qui dispose d'une réserve centralisée?.

\section{Diagnostic}

Bien que les isolats de $C$. diphtheriae soient relativement rares au Canada, l'apport de changements dans les laboratoires de microbiologie clinique de première ligne, comme l'adoption de la MALDITOF MS, pourrait en accroître l'identification. C'est d'ailleurs ce qu'indique un article publié en 2019, dans lequel on fait état d'une augmentation de $1200 \%$ des demandes d'analyse de toxines au Laboratoire national de microbiologie entre 2006 et 2019 (le Laboratoire est passé de 8 à environ 180 analyses par année). La plupart des isolats provenaient d'échantillons cutanés plutôt que respiratoires, et seules $8 \%$ des bactéries en cause étaient toxigènes ${ }^{1}$. Malgré l'augmentation de la détection microbiologique, la diphtérie respiratoire classique reste rare au Canada.

\section{Suivi}

Au Canada, la prise en charge de la diphtérie toxigène par la santé publique est essentielle pour prévenir la transmission de la maladie; elle nécessite une réponse coordonnée (encadré 1) comprenant l'accès facilité à des antitoxines diphtériques ainsi que la recherche et le suivi des contacts (évaluation de leur statut d'immunisation, analyse d'échantillons nasopharyngés et de gorge pour éliminer la possibilité de transmission secondaire, et chimioprophylaxie). Si les premiers échantillons ont donné des résultats positifs, des cultures de suivi doivent être réalisées au moins 24 heures après la fin du traitement antimicrobien, et il faudrait envisager d'élargir la recherche des contacts. Bien que la prise en charge des contacts étroits ne soit généralement nécessaire qu'en cas de diphtérie toxigène, les autorités régionales de santé publique reçoivent souvent les rapports de culture de C. diphtheriae plusieurs jours avant que la toxigénicité de la bactérie soit connue. Ce délai nuit au déploiement rapide de mesures de santé publique si la bactérie s'avère toxigène. Il existe au Royaume-Uni une ligne directrice ${ }^{8}$ formulant des recommandations précises sur la recherche de contacts avant l'obtention des résultats de toxigénicité, en fonction de la gravité du tableau clinique de la personne infectée, des facteurs de risque épidémiologiques augmentant la probabilité de toxigénicité (p. ex., voyage) et du temps estimé pour l'obtention des résultats de laboratoire. La diphtérie non toxigène n'est pas une maladie à déclaration obligatoire au Canada et elle ne nécessite pas de suivi des 
contacts, mais des grappes de diphtérie cutanée non toxigène ont tout de même été bien décrites chez des populations marginalisées, tant au Canada qu'ailleurs ${ }^{9,10}$.

Dans le cas présenté ici, le délai entre l'identification de l'agent pathogène par la culture et la confirmation de la toxigénicité a posé des difficultés de santé publique. Les équipes de soins et de santé publique ont reçu les résultats confirmant la présence de C. diphtheriae et sa toxigénicité une fois la période d'incubation (10 jours) écoulée ${ }^{3}$. Bien qu'aucun des contacts étroits n'ait présenté de symptômes, des interventions de prise en charge ont été déployées pour vérifier si l'un d'entre eux était un porteur asymptomatique pouvant transmettre la maladie ${ }^{8}$. Des études de cas récentes ont fait état d'une transmission secondaire de C. diphtheriae cutanée et respiratoire toxigène à des contacts étroits; cette transmission aurait été découverte par écouvillonnage avant la chimioprophylaxie. La définition de « contacts étroits » englobe les membres du ménage, les personnes ayant eu un contact rapproché ou intime avec la personne infectée et les professionnels de la santé exposés aux sécrétions oropharyngées de cette dernière; elle suit les mêmes principes que celle utilisée en cas de méningococcie invasive ${ }^{11,12}$. Dans une étude de cas sur la diphtérie respiratoire, une transmission subséquente de la maladie par un porteur asymptomatique aurait été repérée à la suite de nouvelles activités de recherche des contacts ${ }^{11}$.

Nous nous sommes aussi questionnés sur la prise en charge des contacts étroits dans le cadre d'un vol international vers le Canada. Comme la méningococcie et la diphtérie se transmettent toutes deux par gouttelettes et que les recommandations concernant la diphtérie sont limitées, nous avons suivi l'approche utilisée au Canada pour la prise en charge des personnes atteintes d'une méningococcie invasive ayant voyagé par avion. Cette approche consiste à rechercher les personnes qui étaient assises de chaque

Encadré 1 : Rôles et responsabilités pour le suivi de la diphtérie toxigène

\section{Secteur}

Soins de santé

(p. ex., hôpitaux, cliniques

communautaires, etc.)

Santé publique (p. ex., échelle régionale, provinciale, nationale)

Laboratoires (p. ex., laboratoires de première ligne, laboratoires de référence provinciaux, Laboratoire national de microbiologie)

\section{Rôles et responsabilités}

- Poser rapidement un diagnostic et traiter sans attendre.

- Aviser rapidement les autorités régionales de santé publique.

- Déployer les mesures appropriées de prévention et de contrôle des infections.

- Aider à l'évaluation des contacts étroits (par le prélèvement d'échantillons nasopharyngés ou de gorge).

- Assurer le suivi des professionnels de la santé qui ont eu des contacts étroits avec la personne infectée et qui doivent être pris en charge.

- Échelle régionale

- Amorcer immédiatement la prise en charge des contacts.

- Effectuer une surveillance locale.

- Appuyer le prélèvement d'échantillons et l'immunisation des contacts.

- Diriger la prise en charge communautaire des contacts

- Diriger la communication avec la communauté, au besoin.

- Échelle provinciale (p. ex., ministère de la Santé, Santé publique Ontario)

- Coordonner l'accès aux antitoxines diphtériques.

- Fournir des conseils ou offrir des consultations aux autorités régionales de santé publique.

- Effectuer une surveillance provinciale.

- Coordonner l'envoi d'un avis à l'Agence de la santé publique du Canada, au besoin.

- Échelle nationale (Agence de la santé publique du Canada)

- Diriger les activités de surveillance nationales.

- Faire le pont avec les gouvernements d'autres pays en vue de la recherche de contacts dans ces pays, au besoin.

- Laboratoires de première ligne

- Analyser les échantillons primaires et faire une première identification de l'agent pathogène.

- Laboratoires de référence provinciaux

- Confirmer les résultats préliminaires; s'il s'agit bel et bien de Corynebacterium diphtheriae, envoyer l'isolat au Laboratoire national de microbiologie.

- Analyser les échantillons primaires; en cas de soupçons, faire une culture pour $C$. diphtheriae (p. ex., contacts d'une personne infectée).

- Laboratoire national de microbiologie

- Faire un test d'amplification en chaîne par polymérase (PCR) pour déterminer si l'isolat est porteur du gène tox. Si c'est le cas, faire un test Elek modifié pour confirmer hors de tout doute que l'isolat produit une toxine diphtérique.

- Tous les laboratoires : fournir des renseignements sur les tests, le temps d'attente pour les résultats et l'interprétation de ceux-ci. 
côté de la personne infectée pendant un vol de plus de 8 heures ${ }^{3,8}$. Cependant, dans le cas qui nous intéresse, la personne infectée était assise à côté de la fenêtre, et son conjoint ou sa conjointe occupait le siège adjacent; il n'y avait donc pas d'autre contact à rechercher. Nous avons également envoyé un avis officiel en Inde par l'intermédiaire de l'Agence de la santé publique du Canada. Aucun autre contact étroit n'y a été identifié.

Ce cas d'infection mortelle nous rappelle que la diphtérie, qui est endémique dans de nombreux pays, peut surgir à tout moment au Canada. Des études de cas récentes venant de pays non endémiques ont d'ailleurs fait état d'infections respiratoires et cutanées toxigènes chez des personnes revenant de voyage, des touristes, des immigrants et des réfugiés ${ }^{11,12}$. En cas de détresse respiratoire chez un patient, les professionnels de la santé devraient garder en tête la possibilité de diphtérie, en particulier chez les voyageurs et les immigrants arrivant de régions endémiques. Il faudrait s'informer du statut vaccinal des nouveaux arrivants et des grands voyageurs pour voir s'il est à jour. La réticence à la vaccination et les déplacements internationaux étant à la hausse, on ne peut fermer les yeux sur l'éventuelle résurgence de maladies depuis longtemps oubliées, comme la diphtérie.

\section{Références}

1. Bernard KA, Pacheco AL, Burdz T, et al. Increase in detection of corynebacterium diphtheriae in Canada: 2006-2019. Can Commun Dis Rep 2019;45:296-301. Accessible ici : www.canada.ca/content/dam/phac-aspc/documents/services/reports -publications/canada-communicable-disease-report-ccdr/monthly-issue/2019-45 /issue-11-november-7-2019/ccdrv45i11a04-eng.pdf (consulté le 14 déc. 2019).
2. Reported cases from 1924 to 2018 in Canada - notifiable diseases on-line [data file]. Ottawa: Public Health Agency of Canada; modified 2020 Aug. 11. Accessible ici : diseases.canada.ca/notifiable/charts?c=pl (consulté le 15 déc. 2019).

3. Infectious diseases protocol - Appendix A: Disease-specific chapters. Chapter: Diphtheria. Toronto: Ministry of Health and Long-Term Care; 2019:1-13. Accessible ici : health.gov.on.ca/en/pro/programs/publichealth/oph_standards/ docs/diphtheria_chapter.pdf (consulté le 15 déc. 2019).

4. Heymann DL, editor. Control of communicable diseases manual. 20th ed. Washington (DC): American Public Health Association; 2014.

5. Diphtheria: for health professionals. Ottawa: Public Health Agency of Canada; modified 2018 June 21 . Accessible ici : www.canada.ca/en/public-health/services /immunization/vaccine-preventable-diseases/diphtheria/health-professionals. html (consulté le 7 déc. 2019).

6. Skogmar S, Tham J. Severe diphtheria with neurologic and myocardial involvement in a Swedish patient: a case report. BMC Infect Dis 2018;18:359.

7. Diphtheria guide for health care professionals. Toronto: Public Health Division, Ministry of Health and Long-Term Care; 2015. Accessible ici : www.health.gov. on.ca/en/pro/publications/disease/pdf/2015-05-22-diphtheria-guide-health-care -professionals.pdf (consulté le 15 déc. 2019).

8. Diphtheria Guidelines Working Group. Public health control and management of diphtheria (in England and Wales): 2015 guidelines. London (UK): Public Health England; 2015:1-47. Accessible ici : assets.publishing.service.gov.uk/ government/uploads/system/uploads/attachment_data/file/774753/Diphtheria _Guidelines_Final.pdf (consulté le 15 déc. 2019).

9. Lowe CF, Bernard KA, Romney MG. Cutaneous diphtheria in the urban poor population of Vancouver, British Columbia, Canada: a 10-year review. J Clin Microbiol 2011;49:2664-6.

10. Dangel A, Berger A, Konrad R, et al. Geographically diverse clusters of nontoxigenic infection, German, 2016-2017. Emerg Infect Dis 2018;24:1239-45.

11. Edwards D, Kent D, Lester C, et al. Transmission of toxigenic Corynebacterium diphtheriae by a fully immunised resident returning from a visit to West Africa, United Kingdom, 2017. Euro Surveill 2018;23:1700681.

12. Jané M, Vidal MJ, Camps $\mathrm{N}$, et al. A case of respiratory toxigenic diphtheria: contact tracing results and considerations following a 30-year disease-free interval, Catalonia, Spain, 2015. Euro Surveill 2018;23:17-00183.

\section{Intérêts concurrents : Aucun déclaré.}

Cet article a été révisé par des pairs.

Les auteurs ont obtenu le consentement du plus proche parent.

Affiliations : Direction générale de la santé publique (Cholewa, Karachiwalla), municipalité régionale de York, Newmarket, Ont.; École de santé publique Dalla Lana (Karachiwalla, Wilson) et Faculté de médecine (Nadarajah) et Département de médecine de laboratoire et de pathologie (Kus), Université de Toronto; Santé publique Ontario (Wilson, Kus), Toronto, Ont.; Département de médecine familiale, École de médecine (Karachiwalla), Université Queen's, Kingston, Ont.; Institut de recherche en services de santé (Wilson), Toronto, Ont.
Collaborateurs : Scott Cholewa et Fareen Karachiwalla ont contribué à la conception du travail. Tous les auteurs ont participé à la rédaction du manuscrit et en ont révisé de façon critique le contenu intellectuel important; ils ont donné leur approbation finale pour la version destinée à être publiée et assument l'entière responsabilité de tous les aspects du travail.

Financement : Le financement de cette étude de cas et du suivi de santé publique provenait de budgets de fonctionnement organisationnels existants.

Propriété intellectuelle du contenu : Il s'agit d'un article en libre accès distribué conformément aux modalités de la licence Creative Commons Attribution (CC BY-NC-ND 4.0), qui permet l'utilisation, la diffusion et la reproduction dans tout médium à la condition que la publication originale soit adéquatement citée, que l'utilisation se fasse à des fins non commerciales (c.-à-d., recherche ou éducation) et qu'aucune modification ni adaptation n'y soit apportée. Voir : https://creativecommons.org/ licenses/by-nc-nd/4.0/deed.fr.

Remerciements : Les auteurs remercient les personnes suivantes pour leur contribution : la $D^{\text {re }}$ Michelle Murti, Santé publique Ontario; Martina Cuillerier, Thomas Lo, Andrea Main, Marjolyn Pritchard et Candace Wong, Santé publique de la région de York; la $\mathrm{D}^{\text {re }}$ Deborah Yamamura, Centre des sciences de la santé de Hamilton; Kathryn Bernard, Laboratoire national de microbiologie; personnel du laboratoire du Centre des sciences de la santé de Hamilton, du laboratoire de Santé publique Ontario et du Laboratoire national de microbiologie.

Correspondance : Scott Cholewa, scott.cholewa@york.ca 